\title{
Nanotechnologies for Curcumin: An Ancient Puzzler Meets Modern Solutions
}

\author{
Shengpeng Wang, Miao Tan, Zhangfeng Zhong, Meiwan Chen, and Yitao Wang \\ State Key Laboratory of Quality Research in Chinese Medicine, Institute of Chinese Medical Sciences, University of Macau, \\ Avenve Padre Tomas Pereira S.J., Taipa 999078, Macau \\ Correspondence should be addressed to Meiwan Chen, chenmeiwan81@163.com and Yitao Wang, pktwhkmo@163.com
}

Received 4 July 2011; Accepted 18 July 2011

Academic Editor: Chunyi Zhi

Copyright ( $\odot 2011$ Shengpeng Wang et al. This is an open access article distributed under the Creative Commons Attribution License, which permits unrestricted use, distribution, and reproduction in any medium, provided the original work is properly cited.

\begin{abstract}
Curcumin, a low-molecular-weight natural polyphenol mainly found in the plant Curcuma longa (turmeric), is widely used as a food colorant and as a potential protective agent against several chronic diseases including cancer, HIV-infection, neurological, cardiovascular, and skin diseases. Moreover, evidences from long-term use process and preclinical trials have demonstrated low toxicity of curcumin, even at relatively high doses. However, it has been well known that the application of curcumin was limited owing to its water insolubility, instability, and poor bioavailability. For decades, many attempts have been made to compensate for these disadvantages, with the development of improved delivery platforms as the feasible approaches. The past ten years witnessed the encouraging progress in the use of nanoscale drug delivery systems on curcumin such as loading curcumin into liposomes or nanoparticles, forming self-microemulsifying drug delivery systems (SMEDDS), cyclodextrin inclusions, and solid dispersions, as well as the latest reported technologies such as nadodisks and nanotubes. This paper summarizes the recent works on the design and development of nanoscale delivery systems of curcumin, with the goal of harnessing the true difficulties of this multifunctional agent in the clinical arena.
\end{abstract}

\section{Introduction}

Curcumin (Figure 1), an active constituent mainly derived from Curcuma longa (turmeric), is a natural yellow-orange polyphenol which has been used for its medicinal benefits for centuries $[1,2]$. Curcumin was firstly extracted in impure form in 1815, then in 1870 the pure crystalline state was prepared. Almost three decades later, its composition was finally elucidated as 1, 6-heptadiene-3, 5-dione-1, 7-bis-(4hydroxy-3-methoxyphenyl)-(1E, 6E) [3-6]. In China, curcumin has been used as a part of herbal medicine for centuries to alleviate throbbing pain in the chest and hypochondriac region, mass in abdomen, and pain of the shoulder due to win-cold or traumatic injuries. The accumulating of experimental and clinical evidences indicates that curcumin has a variety of pharmacological activities, such as antitumor, antiinflammatory, antivirus, antioxidation, anti-HIV, and low toxicity [7-11].
However, good things never come easy. Applicational advancement of curcumin has been hindered by its water insolubility, degradation at alkaline $\mathrm{pH}$, and photodegradation and thus extremely low bioavailability in both vascular and oral administration $[12,13]$. Therefore, many approaches have been investigated, including synthetic analogues, chemical modification to prodrugs, combined with other dietary components and using nanoscale drug delivery systems to overcome deficiencies.

Among these methods, nanoscale drug delivery systems have become a source of concern for many researchers in the domain of tradional Chinese medicine (TCM). Many successful examples of the combination of nanotechnology and TCM have been reported. Due to the surface effect, small size effect, quantum size effect, and quantum tunnel effect of nanoparticles, nanoscaled TCM has produced some new outcomes. For instance, xionghuang (realgar), a famous TCM, has been identified as an effective antitumor agent. When 
<smiles>COc1cc(/C=C/C(=O)CC(=O)/C=C/c2ccc(O)c(OC)c2)ccc1O</smiles>

FIgURE 1: Chemical structure of curcumin.

fabricated into nanoscale, the apoptosis rate of the tumor cells was markedly increased [14]. Furthermore, nanotechnologies disrupt the cell membrane and thus release the trapped materials. Take Ganoderma lucidum (Lingzhi) for example, the immune function could be evidently enhanced after nanoscale processes [15].

Consequently, a good understanding of nanotechnologies is necessary for the advancement of curcumin with higher efficacy. To date, many studies have focused on loading curcumin into liposomes or nanoparticles, forming selfmicroemulsifying drug delivery systems (SMEDDS), cyclodextrin inclusions, and solid dispersions [16-18], and much progress has been made in the past ten years. The purpose of this paper is to provide an updated summary of the applications of novel delivery systems of curcumin.

\section{Liposomes}

Liposomes (Figure 2) are closed spherical vesicles consisting of a lipid bilayer that encapsulates an aqueous phase in which drugs can be stored [19]. Possessing the advantages of high biocompatibility, easy preparation, chemical versatility, and simple modulation of their pharmacokinetic properties by changing the chemical composition of the bilayer components, liposomes as a drug delivery system have been used to improve the therapeutic activity and safety of drugs for many years [20].

By the same token, liposomes have found wide application in ameliorating curcumin's bioavailability and efficacy; indeed, various modifications of liposomal curcumin have been developed such as polymeric conjugation on the liposome surface to acquire better clinical outcomes [4, 17-26]. The exploration disclosed an effective method for the preparation of curcumin-loaded liposomes using sonication at an average size of 100-150 nm [22]. Exclusion chromatography was used to remove the unentrapped curcumin. In vitro tests showed that treatment of liposomal curcumin $(5-10 \mu \mathrm{M})$ for $24-48 \mathrm{~h}$ at $37^{\circ} \mathrm{C}$ resulted in at least a $70-80 \%$ inhibition of cellular proliferation without affecting their viability, which was approximately 10 -fold higher versus free curcumin.

\section{Nanoparticles}

Nanoparticles (NPs), which are particles ranging in size from 1 to $100 \mathrm{~nm}$, possess distinct physical and chemical properties that can be exploited for drug delivery [19]. Encapsulating drugs within NPs can improve the solubility and pharmacokinetics of drugs and sometimes enable targeting and slow release. Generally, nanoparticles' carrier materials can be divided into synthetic biodegradable high molecular polymers (polyvinyl alcohol, polylactic acid, etc.) and natural polymers (proteins, polysaccharides, etc.) [27]. Among the various nanodrug systems, polymer NPs, solid lipid NPs, magnetic NPs, polymer micelles, and albumin NPS are the five ones that are widely applied and intensively studied on curcumin.

3.1. Polymer Nanoparticles. Due to the small size and excellent biocompatibility, nanosized polymer nanoparticles can circulate in the bloodstream for a longer time; thus, specific therapy can be achieved [28]. The widely researched synthetic polymers include chitosan $[29,30]$, poly(D,L-lactideco-glycolide) (PLGA) [31-33], and PEG. In addition, other carrier materials such as poly(butyl)cyanoacrylate [34], silk fibroin [35], N-isopropylacrylamide (NIPAAM) [36], and hydrophobically modified starch [37] have also shown a high potential for solving the problems of curcumin.

PLGA and a stabilizer polyethylene glycol- (PEG-)5000 were employed to form a nanoparticulate curcumin formulation with $97.5 \%$ efficiency encapsulation and $80.9 \mathrm{~nm}$ particle diameter [32]. The result of an in vitro experiment demonstrated that curcumin-loaded PLGA NPs enhanced cellular uptake and increased bioactivity in inducing apoptosis and suppressing proliferation of tumor cells. The nanoparticulate formulation also showed better bioavailability and had a longer half-life than free curcumin in mice. Moreover, polymers can be combined to form copolymers, which could be a promising drug carrier. For example, the PLGAPEG-PLGA triblock copolymers prepared using the solventdialysis method could encapsulate hydrophobic curcumin, which indicated good pharmacokinetic profiles [38].

3.2. Solid Lipid Nanoparticles (SLNs). SLNs are made of natural or synthetic lipid or lipoid, such as lecithin and triglycerides, which are solid at human physiological temperature [39]. SLNs present many potential advantages. For example, they protect labile compounds from chemical degradation, provide sustainedrelease to improve the availability of the drug, and target the effect to improve the efficiency of the drugs [40].

Kakkar et al. [41] prepared curcumin-loaded solid lipid nanoparticles (C-SLNs), with an average particle size of $134.6 \mathrm{~nm}$ and a total drug content of $92.33 \pm 1.63 \%$, using the microemulsification technique. In vivo studies demonstrated significant improvement in bioavailability after administration of C-SLNs at all the doses other than free solubilized curcumin. In another study, transferring-mediated solid lipid nanoparticles containing curcumin were recently fabricated [42], and the potential of enhancing the anticancer effect of curcumin in breast cancer cells in vitro was confirmed.

3.3. Magnetic Nanoparticles. Magnetic drug targeting, in which a drug is conjugating with a magnetic material under the action of the external magnetic field, is an important drug delivery system. Drug-loaded magnetic nanoparticles 


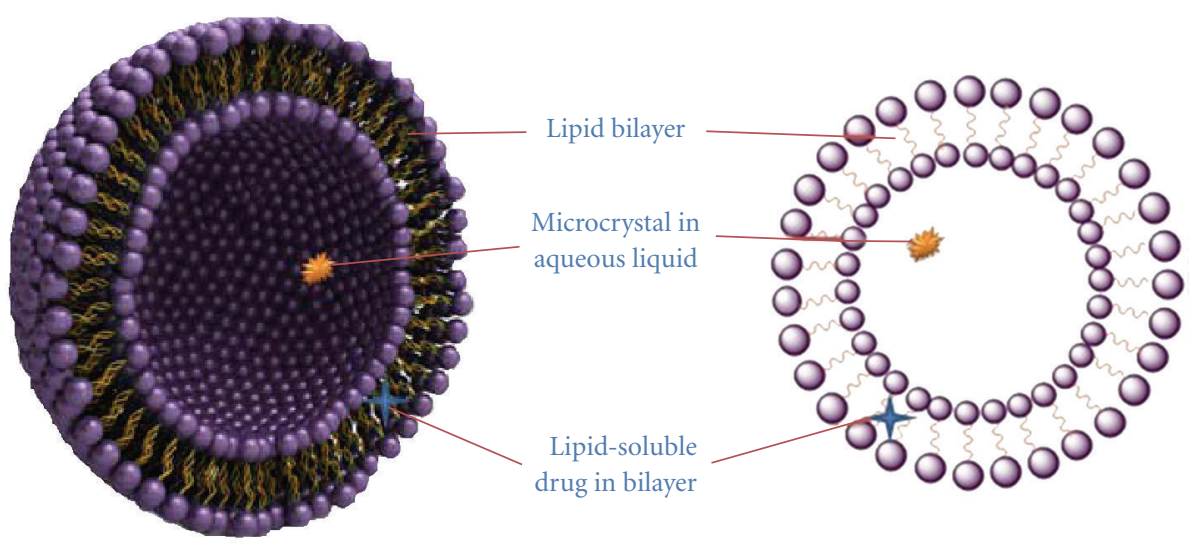

FIGURE 2: Structure of liposomes.

can accumulate in target tissue areas under the action of the external magnetic field; the drug then releases from the particles in a controllable way [43].

A nanosized magnetofluorescent water-dispersible $\mathrm{Fe}_{3} \mathrm{O}_{4}$-curcumin conjugate with chitosan or oleic acid as its outer shell and entrapped curcumin was designed [44]. The $\mathrm{Fe}_{3} \mathrm{O}_{4}$-curcumin conjugate exhibited a high-loading cellular uptake that was distinctly observed by magnetic and fluorescent methods and was also shown to be a good candidate for a dual (optical and magnetic) imaging probe.

3.4. Polymer Micelles. Another feasible approach to figuring out a way to solve the poor solubility, stability, and bioavailability of curcumin is to encapsulate it within micelles $[45,46]$. Recently, polymeric micelles have gained attention as an excellent delivery system for poorly water-soluble drugs due to their high drug-loading capacity, high watersolubility, low toxicity, and appropriate size $(<200 \mathrm{~nm})$ for long circulation in the blood $[45,47,48]$.

For example, Leung et al. [49] investigated encapsulation of curcumin in cationic micelles composed of surfactants cetyl trimethylammonium bromide (CTAB) or dodecyl trimethylammonium bromide (DTAB). Data showed that alkaline hydrolysis of curcumin is greatly curbed with the aid of either CTAB or DTAB micelles. Along the same lines, amphiphilic block copolymer micelles of poly(ethylene oxide)b-poly(epsilon-caprolactone) (PEO-PCL) as vehicles for curcumin were also reported [50]. The author believed that PEO-PCL micelles as an injectable formulation can effectively solubilize, stabilize, and control the release of curcumin.

3.5. Albumin Nanoparticles. The possibility of loading a fair amount of active components of poor aqueous solubility is an issue of common concern. Albumin is the body's natural carrier of hydrophobic molecules, such as fatty acids, hormones, and fat-soluble vitamins [51]. Albumin has been extensively investigated as a drug carrier due to its nontoxicity and nonimmunogenicity [52]. Furthermore, albumin possesses good solubility in both water and ethanol, which provide a specific capacity for enhancing the solubility of curcumin. Novel curcumin-loaded human serum albumin nanoparticles (CCM-HSA-NPs) with a narrow size distribution in the 130-150 nm range were prepared [52].

The CCM-HSA-NPs showed a much greater water solubility (300-fold) than free curcumin and experienced negligible activity loss during storage [52]. The amounts of curcumin in tumors, vascular endothelial cell binding of curcumin, and transport of curcumin across a vascular endothelial cell monolayer after treatment with CCM-HSA-NPs were increased 14-, 5.5-, and 7.7-fold beyond that achieved by free curcumin. Furthermore, in vivo antitumor tests revealed that CCM-HSA-NPs had a better therapeutic effect than free curcumin without leading to toxicity.

\section{Microsphere and Microcapsule}

When a pharmaceutical agent is encapsulated within or dispersed in polymer materials, drug safety and efficacy can be greatly improved and new therapies are possible. Given the protection and selective permeation properties and organtargeted release feasibility, microspheres and microcapsules are broadly applied not only in the food-making and cosmetics industry, but also in pharmaceutical fields such as drug delivery recently. Thus, a series of natural active ingredients, such as ptothecin, zedoary oil, rutin, and andrographolide, have been made into microspheres or microcapsules [28].

Many researchers have made some attempts to deliver curcumin by microspheres or microcapsules [53-57]. The fabrication of microcapsules using a layer-by-layer (LbL) approach with curcumin demonstrated that curcumin can be accumulated in hollow microcapsules with polyelectrolyte multilayer shells (PSS/PEI) 6 [55]. Bioactivity of the released drug is evaluated by cytotoxicity studies using L929 cell lines. The new formulation was found to be cytocompatible while the extract of capsules loaded with curcumin showed severe cytotoxicity on the mouse fibroblast cell, indicating that the released curcumin is active. In addition, the high stability of polyelectrolyte microcapsules in the aqueous medium indicates that these drug carriers are suitable for drug delivery applications. 


\section{Microemulsion}

Microemulsions are isotropic dispersions consisting of oil and water stabilized by an interfacial film of surfactant molecules, typically in conjunction with a cosurfactant. Due to their extremely small emulsion droplet sizes in the range of 50 to $200 \mathrm{~nm}$, which are much smaller than conventional emulsions ranging from 1 to $100 \mu \mathrm{m}$, microemulsions as a lipid-based delivery system possess many advantages, including high solubilization potential, thermodynamic stability, improved drug dissolution and surfactant-induced permeability enhancement $[58,59]$. Among various drug delivery systems, the microemulsion system is considered to be an ideal alternative for hydrophobic drugs such as curcumin.

A number of studies have recently been published about the preparation methods, evaluation and pharmacokinetics profiles of curcumin microemulsions [16, 58, 60-62]. Using medium chain triacylglycerols as the oil and Tween 20 as emulsifier, $\mathrm{O} / \mathrm{W}$ emulsions with mean droplet sizes ranging from $618.6 \mathrm{~nm}$ to $79.5 \mathrm{~nm}$ have been successfully prepared using high-speed homogenization at 24,000 rpm and highpressure homogenization at 1500 bar [58]. A 12-O-tetradecanoylphorbol-13-acetate-induced [63] mouse ear inflammation model was built to estimate the activity of curcumin nanoemulsion. A $43 \%$ or $85 \%$ inhibition effect of edema of mouse ear exists for $618.6 \mathrm{~nm}$ and $79.5 \mathrm{~nm} 1 \%$ curcumin nanoemulsions, respectively, while the oral administration of $1 \%$ curcumin in Tween 20 water solution shows little or no inhibition effect.

Self-microemulsifying drug delivery systems (SMEDDS) have recently emerged as one of the most interesting approaches to improving the solubility, dissolution, and oral absorption for poorly water-soluble drugs [64]. Setthacheewakul et al. [60] successfully developed a new SMEDDS to improve the solubility and oral absorption of curcumin. A simplex lattice experiment design was used to optimize the formulation of SMEDDS: $57.5 \%$ surfactant (emulsifier OP: Cremophor EL = 1:1), 30.0\% cosurfactant (PEG 400), and $12.5 \%$ oil (ethyl oleate), which showed a significant improvement of solubility of curcumin $(21 \mathrm{mg} / \mathrm{g})$. The results of an oral absorption experiment in mice demonstrated that SMEDDS could obviously increase the oral absorption of curcumin compared with its suspension.

\section{Cyclodextrin Inclusion}

Cyclodextrins (Figure 3) are cyclic oligosaccharides with a hydrophilic outer surface and lipophilic central cavity. Three types of cyclodextrins exist: $\alpha$-cyclodextrin, $\beta$-cyclodextrin, and $\gamma$-cyclodextrin which are composed of six, seven, and eight $\alpha$-(1,4)-linked glycosyl units, respectively [65]. Due to the advantages of accessibility and lowest-price, $\beta$-cyclodextrin is generally the most useful. Possessing a special ability of enabling drugs to increase water solubility, reduce bitterness, enhance stability, and improve bioavailability, cyclodextrins have been used extensively in pharmaceutical research and development $[66,67]$.

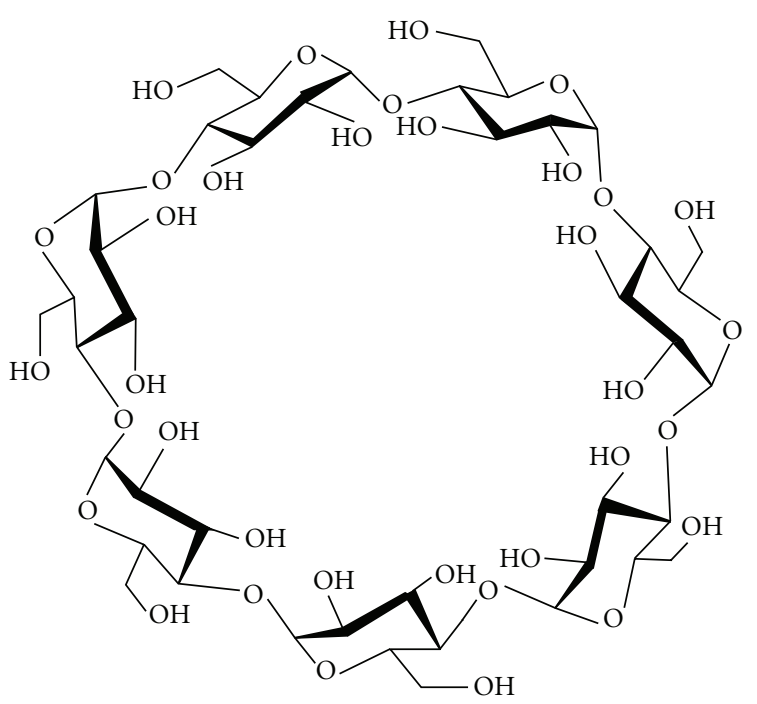

Figure 3: Chemical structure of $\beta$-cyclodextrin.

Recently, a growing body of experimental evidence has suggested potential significance in exploring the cyclodextrin carrier properties for the delivery of curcumin [68-72].

In order to explore the cyclodextrin carrier properties for the delivery of curcumin, a self-assembly of $\beta$-cyclodextrin and curcumin was prepared via inclusion complexation using a solvent evaporation technique [70]. This study demonstrated that curcumin was efficiently encapsulated in $\beta$-cyclodextrin cavities and the developed self-assemblies were confirmed by spectroscopy (FTIR, ${ }^{1} \mathrm{H}$ NMR), thermal studies (DSC and TGA), X-ray diffraction, and microscopic studies (SEM and TEM). In addition, the $\beta$-cyclodextrin-curcumin inclusion complex showed an improved uptake in DU145 cancer cells and greater potent therapeutic efficacy in prostate cancer cells compared to free curcumin.

In addition, a novel cyclodextrin complex of curcumin (CDC) was manufactured through a $\mathrm{pH}$ shift method using a highly alkaline solution for curcumin dissolution and contacting with hydroxypropyl- $\gamma$-cyclodextrin $(\mathrm{HP} \gamma \mathrm{CD}$ ) [69]. These aqueous curcumin formulations have about 100-fold greater curcumin concentration than previously described cyclodextrin complexes of curcumin, and their stability is remarkably improved. In vitro tests demonstrated that CDC had more potential compared with free curcumin for cellular uptake and for antiproliferative and anti-inflammatory activities.

\section{Solid Dispersion}

In 1971, solid dispersion was defined as a dispersion of one or more active ingredients in an inert carrier or matrix in the solid state prepared by the melting (fusion), solvent, or melting-solvent methods [73]. In recent years, increasing attention has focused on solid dispersion systems, finding that they can increase bioavailability of poorly water-soluble drugs such as curcumin [74-77]. 

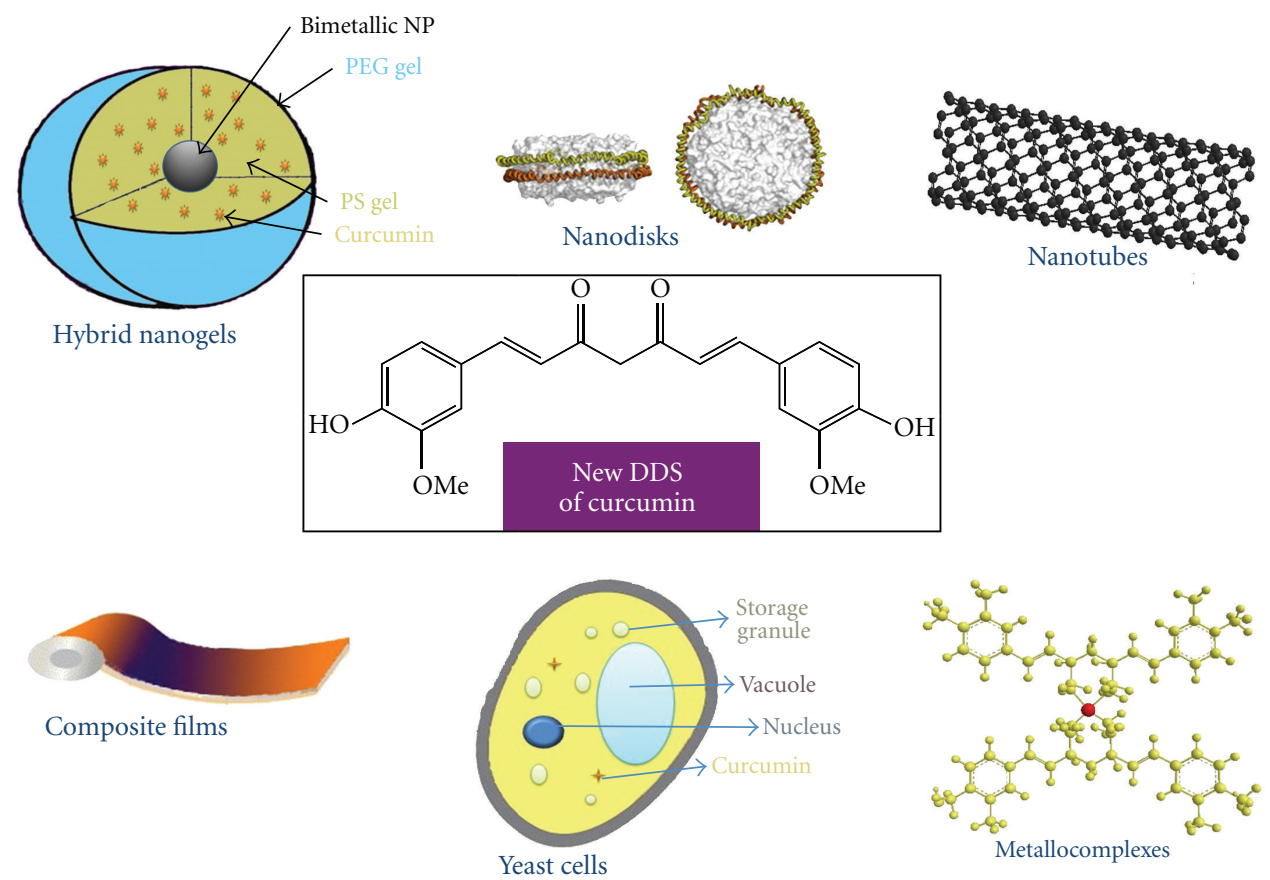

FIGURE 4: Most recent DDS of curcumin.

Crystal and amorphous solid dispersions have also been designed by wet-milling and subsequent freeze-drying with the aim of improving physicochemical and pharmacokinetic profiles of curcumin [78]. The mean particle size of curcumin was identified to be ca. $250 \mathrm{~nm}$, with a small polydispersity index in crystal solid dispersion, compared to crystalline curcumin, the authors demonstrated that the rapid dissolution/ dispersion behavior and oral bioavailability increased by 12 and 16-fold, respectively.

\section{Miscellaneous Novel Delivery Systems}

Researchers have also tried other ways to improve the biological activity of curcumin (Figure 4). The application of these techniques offered new research direction and a visual field for solving the limitations of curcumin.

A class of unique hybrid nanogels was designed to improve the therapeutic efficacy of curcumin [79]. These formulations with high drug-loading yields are claimed to be well suited for in vivo studies and clinical trials. Several metallocomplexes of curcumin have been synthesized, characterized, and evaluated for various biological activities. Barik et al. [63] reported that a $1: 1$ complex of copper with curcumin exhibited SOD activity and in vitro antioxidant activity. Nadodisks (NDs) represent a potentially novel means to deliver curcumin effectively. NDs are disk-shaped phospholipid bilayers whose edge is stabilized by apolipoproteins, they may have particular in vivo therapeutic applications due to their nanoscale size and ability to solubilize curcumin [80]. Paramera et al. [68] evaluated the stability of yeast cell-encapsulated curcumin in regard to environmental factors such as light, humidity, and heat. They found that yeast cells significantly protected curcumin from the detrimental effects of light, as well as from oxidative degradation at elevated relative humidity.

In addition, curcumin-loaded films were prepared using biodegradable ploymer (PLGA) and in vitro tests indicated that the curcumin-loaded films have better anticoagulative effects than PLGA. Recently, a combination of nanotubes and curcumin also are in application in the chemical industry [81].

\section{Problems and Prospects}

Since the discovery of the enhanced drug permeation and retention effect in the 1980s, the advancements in the nanotechnology are widely penetrating to the field of life sciences. We should be thankful that the nanotechnologies bring a real innovation for cracking many medical science hard nuts. An ideal nanoscale agent enables drug arrivals and acts preferentially at the selected target, hence the therapeutic effect could be markedly improved. Undoubtedly, curcumin, an excellent representative derived from traditional Chinese medicine, has been proven effective in long-term use and preclinical trials. Therefore, it is of great significance to overcome the current limitations of curcumin. In past decades, researchers have made real strides in the inhibition of nanotechnologies 
on curcumin, but we should also be soberly aware that some problems still remain.

Curcumin liposome formulations are known to improve bioavailability and efficacy and reduce toxicity, but no tissue specificity is associated with the liposomes. After entering the body, liposomes are rapidly taken up by the reticuloendothelial system in the liver and spleen, leading to a short circulation time. Therefore, developing effective modifications of liposomes such as polymeric conjugation on liposome surface becomes essential to acquire better clinical outcomes. Furthermore, efficacious and simple as they are, microemulsions also contain multiple surfactants, which leads to inevitable toxicity. Thus the choice of the surfactants is critical for the formulation of microemulsions. Additionally, most of the proposed formulations were derived from synthetic polymers: long-period employments of these excipients can achieve temporary increased solubility and bioavailability, but potential harms may occur. The search for excipients has been an important part of the current pharmaceutical research and this trend seems irresistible.

Taken together, there is no doubt that development of novel delivery systems of curcumin with better curative effects will be critical for future development of curcumin as a therapeutic agent. We strongly believe that a desired preparation will not only load and keep the drug stable, but will also exactly deliver the drug to candidate cells to enhance the therapeutic effect and reduce the toxicity to normal cells. This is an arduous and complex job: only by multidisciplinary cooperation can we bring this promising natural medicine to the forefront of therapeutic agents for cancers and other diseases.

\section{Acknowledgment}

This study was supported by the Research Fund of the University of Macau (MYRG 208 (Y1-L4)-ICMS11-WYT).

\section{References}

[1] R. M. Srivastava, S. Singh, S. K. Dubey, K. Misra, and A. Khar, "Immunomodulatory and therapeutic activity of curcumin," International Immunopharmacology, vol. 11, no. 3, pp. 331341, 2010.

[2] R. Li, C. Xiang, M. Ye, H. F. Li, X. Zhang, and D. A. Guo, "Qualitative and quantitative analysis of curcuminoids in herbal medicines derived from Curcuma species," Food Chemistry, vol. 126, no. 4, pp. 1890-1895, 2011.

[3] R. F. Tayyem, D. D. Heath, W. K. Al-Delaimy, and C. L. Rock, "Curcumin content of turmeric and curry powders," Nutrition and Cancer, vol. 55, no. 2, pp. 126-131, 2006.

[4] N. K. Gupta and V. K. Dixit, "Development and evaluation of vesicular system for curcumin delivery," Archives of Dermatological Research, vol. 303, no. 2, pp. 89-101, 2010.

[5] S. Shishodia, G. Sethi, and B. B. Aggarwal, "Curcumin: getting back to the roots," Annals of the New York Academy of Sciences, vol. 1056, pp. 206-217, 2005.

[6] S. Bisht and A. Maitra, "Systemic delivery of curcumin: 21st century solutions for an ancient conundrum," Current Drug Discovery Technologies, vol. 6, no. 3, pp. 192-199, 2009.
[7] K.-Y. Yang, L.-C. Lin, T.-Y. Tseng, S.-C. Wang, and T.-H. Tsai, "Oral bioavailability of curcumin in rat and the herbal analysis from Curcuma longa by LC-MS/MS," Journal of Chromatography B, vol. 853, no. 1-2, pp. 183-189, 2007.

[8] R. A. Sharma, A. J. Gescher, and W. P. Steward, "Curcumin: the story so far," European Journal of Cancer, vol. 41, no. 13, pp. 1955-1968, 2005.

[9] J. S. Jurenka, "Anti-inflammatory properties of curcumin, a major constituent of Curcuma longa: a review of preclinical and clinical research," Alternative Medicine Review, vol. 14, no. 2, pp. 141-153, 2009.

[10] P. Anand, C. Sundaram, S. Jhurani, A. B. Kunnumakkara, and B. B. Aggarwal, "Curcumin and cancer: an "old-age" disease with an "age-old” solution," Cancer Letters, vol. 267, no. 1, pp. 133-164, 2008.

[11] R. K. Maheshwari, A. K. Singh, J. Gaddipati, and R. C. Srimal, "Multiple biological activities of curcumin: a short review," Life Sciences, vol. 78, no. 18, pp. 2081-2087, 2006.

[12] H. Tang, C. J. Murphy, B. Zhang et al., "Curcumin polymers as anticancer conjugates," Biomaterials, vol. 31, no. 27, pp. 71397149, 2010.

[13] P. Anand, A. B. Kunnumakkara, R. A. Newman, and B. B. Aggarwal, "Bioavailability of curcumin: problems and promises," Molecular Pharmaceutics, vol. 4, no. 6, pp. 807-818, 2007.

[14] Y. Yue, Y. Juan, C. Jing et al., "The apoptosis of lung cancer A549 cell induced by realgar and realgar nanoparticle," AsiaPacific Traditional Medicine, vol. 6, no. 6, pp. 8-11, 2010.

[15] X. Caiju, Z. Ronghua, M. Jia et al., "Effect of nano spore powder of Ganoderma lucidum on immune function of mice," Pharmacology and Clinics of Chinese Materia Medica, vol. 21, no. 5, pp. 36-38, 2005.

[16] J. Cui, B. Yu, Y. Zhao et al., "Enhancement of oral absorption of curcumin by self-microemulsifying drug delivery systems," International Journal of Pharmaceutics, vol. 371, no. 1-2, pp. 148-155, 2009.

[17] S. Mourtas, M. Canovi, C. Zona et al., "Curcumin-decorated nanoliposomes with very high affinity for amyloid- $\beta 1-42$ peptide," Biomaterials, vol. 32, no. 6, pp. 1635-1645, 2011.

[18] W. Tiyaboonchai, W. Tungpradit, and P. Plianbangchang, "Formulation and characterization of curcuminoids loaded solid lipid nanoparticles," International Journal of Pharmaceutics, vol. 337, no. 1-2, pp. 299-306, 2007.

[19] Y. Malam, M. Loizidou, and A. M. Seifalian, "Liposomes and nanoparticles: nanosized vehicles for drug delivery in cancer," Trends in Pharmacological Sciences, vol. 30, no. 11, pp. 592599, 2009.

[20] E. Terreno, D. Delli Castelli, C. Cabella et al., "Paramagnetic liposomes as innovative contrast agents for magnetic resonance (MR) molecular imaging applications," Chemistry and Biodiversity, vol. 5, no. 10, pp. 1901-1912, 2008.

[21] D. Wang, M. S. Veena, K. Stevenson et al., "Liposome-encapsulated curcumin suppresses growth of head and neck squamous cell carcinoma in vitro and in xenografts through the inhibition of nuclear factor $\kappa \mathrm{B}$ by an AKT-independent pathway," Clinical Cancer Research, vol. 14, no. 19, pp. 6228-6236, 2008.

[22] R. L. Thangapazham, A. Puri, S. Tele, R. Blumenthal, and R. K. Maheshwari, "Evaluation of a nanotechnology-based carrier for delivery of curcumin in prostate cancer cells," International Journal of Oncology, vol. 32, no. 5, pp. 1119-1123, 2008.

[23] L. Ji, J. Yunyun, W. Jun, F. Guorong, W. Yutian, and Z. Chuan, "A rapid and simple HPLC method for the determination of 
curcumin in rat plasma: assay development, validation and application to a pharmacokinetic study of curcumin liposome," Biomedical Chromatography, vol. 23, no. 11, pp. $1201-$ 1207, 2009.

[24] W. Hong, D.-W. Chen, X.-L. Zhao, M.-X. Qiao, and H.-Y. $\mathrm{Hu}$, "Preparation and study in vitro of long-circulating nanoliposomes of curcumin," China Journal of Chinese Material Medica, vol. 33, no. 8, pp. 889-892, 2008.

[25] A. Kunwar, A. Barik, R. Pandey, and K. I. Priyadarsini, "Transport of liposomal and albumin loaded curcumin to living cells: an absorption and fluorescence spectroscopic study," Biochimica et Biophysica Acta, vol. 1760, no. 10, pp. 1513-1520, 2006.

[26] C. Chen, T. D. Johnston, H. Jeon et al., "An in vitro study of liposomal curcumin: stability, toxicity and biological activity in human lymphocytes and Epstein-Barr virus-transformed human B-cells," International Journal of Pharmaceutics, vol. 366, no. 1-2, pp. 133-139, 2009.

[27] M. Wang and M. Thanou, "Targeting nanoparticles to cancer," Pharmacological Research, vol. 62, no. 2, pp. 90-99, 2010.

[28] D. C. Li, X. K. Zhong, Z. P. Zeng et al., "Application of targeted drug delivery system in Chinese medicine," Journal of Controlled Release, vol. 138, no. 2, pp. 103-112, 2009.

[29] A. Anitha, S. Maya, N. Deepa et al., "Efficient water soluble O-carboxymethyl chitosan nanocarrier for the delivery of curcumin to cancer cells," Carbohydrate Polymers, vol. 83, no. 2, pp. 452-461, 2011.

[30] R. K. Das, N. Kasoju, and U. Bora, "Encapsulation of curcumin in alginate-chitosan-pluronic composite nanoparticles for delivery to cancer cells," Nanomedicine, vol. 6, no. 1, pp. e153e160, 2010.

[31] M. M. Yallapu, B. K. Gupta, M. Jaggi, and S. C. Chauhan, "Fabrication of curcumin encapsulated PLGA nanoparticles for improved therapeutic effects in metastatic cancer cells," Journal of Colloid and Interface Science, vol. 351, no. 1, pp. 19-29, 2010.

[32] P. Anand, H. B. Nair, B. Sung et al., "Design of curcuminloaded PLGA nanoparticles formulation with enhanced cellular uptake, and increased bioactivity in vitro and superior bioavailability in vivo," Biochemical Pharmacology, vol. 79, no. 3, pp. 330-338, 2010.

[33] J. Shaikh, D. D. Ankola, V. Beniwal, D. Singh, and M. N. V. R. Kumar, "Nanoparticle encapsulation improves oral bioavailability of curcumin by at least 9-fold when compared to curcumin administered with piperine as absorption enhancer," European Journal of Pharmaceutical Sciences, vol. 37, no. 3-4, pp. 223-230, 2009.

[34] R. Mulik, K. Mahadik, and A. Paradkar, "Development of curcuminoids loaded poly(butyl) cyanoacrylate nanoparticles: physicochemical characterization and stability study," European Journal of Pharmaceutical Sciences, vol. 37, no. 3-4, pp. 395-404, 2009.

[35] V. Gupta, A. Aseh, C. N. Ríos, B. B. Aggarwal, and A. B. Mathur, "Fabrication and characterization of silk fibroin-derived curcumin nanoparticles for cancer therapy," International Journal of Nanomedicine, vol. 4, pp. 115-122, 2009.

[36] S. Bisht, G. Feldmann, S. Soni et al., "Polymeric nanoparticleencapsulated curcumin ("nanocurcumin"): a novel strategy for human cancer therapy," Journal of Nanobiotechnology, vol. 5, article 3, 2007.

[37] H. Yu and Q. Huang, "Enhanced in vitro anti-cancer activity of curcumin encapsulated in hydrophobically modified starch," Food Chemistry, vol. 119, no. 2, pp. 669-674, 2010.
[38] Z. Song, R. Feng, M. Sun et al., "Curcumin-loaded PLGAPEG-PLGA triblock copolymeric micelles: preparation, pharmacokinetics and distribution in vivo," Journal of Colloid and Interface Science, vol. 354, no. 1, pp. 116-123, 2011.

[39] J. Pardeike, A. Hommoss, and R. H. Müller, "Lipid nanoparticles (SLN, NLC) in cosmetic and pharmaceutical dermal products," International Journal of Pharmaceutics, vol. 366, no. 12, pp. 170-184, 2009.

[40] R. R. Zhu, L. L. Qin, M. Wang et al., "Preparation, characterization, and anti-tumor property of podophyllotoxinloaded solid lipid nanoparticles," Nanotechnology, vol. 20, no. 5, Article ID 055702, 2009.

[41] V. Kakkar, S. Singh, D. Singla, and I. P. Kaur, "Exploring solid lipid nanoparticles to enhance the oral bioavailability of curcumin," Molecular Nutrition and Food Research, vol. 55, no. 3, pp. 495-503, 2011.

[42] R. S. Mulik, J. Mönkkönen, R. O. Juvonen, K. R. Mahadik, and A. R. Paradkar, "Transferrin mediated solid lipid nanoparticles containing curcumin: enhanced in vitro anticancer activity by induction of apoptosis," International Journal of Pharmaceutics, vol. 398, no. 1-2, pp. 190-203, 2010.

[43] Y. Yang, J. S. Jiang, B. Du, Z. F. Gan, M. Qian, and P. Zhang, "Preparation and properties of a novel drug delivery system with both magnetic and biomolecular targeting," Journal of Materials Science, vol. 20, no. 1, pp. 301-307, 2009.

[44] L. D. Tran, N. M. T. Hoang, T. T. Mai et al., "Nanosized magnetofluorescent $\mathrm{Fe} 3 \mathrm{O} 4$-curcumin conjugate for multimodal monitoring and drug targeting," Colloids and Surfaces A, vol. 371, no. 1-3, pp. 104-112, 2010.

[45] A. Sahu, U. Bora, N. Kasoju, and P. Goswami, "Synthesis of novel biodegradable and self-assembling methoxy poly(ethylene glycol)-palmitate nanocarrier for curcumin delivery to cancer cells," Acta Biomaterialia, vol. 4, no. 6, pp. 1752-1761, 2008.

[46] M. O. Iwunze, "Binding and distribution characteristics of curcumin solubilized in CTAB micelle," Journal of Molecular Liquids, vol. 111, no. 1-3, pp. 161-165, 2004.

[47] G. S. Kwon and T. Okano, "Polymeric micelles as new drug carriers," Advanced Drug Delivery Reviews, vol. 21, no. 2, pp. 107-116, 1996.

[48] K. M. Huh, S. C. Lee, Y. W. Cho, J. Lee, J. H. Jeong, and K. Park, "Hydrotropic polymer micelle system for delivery of paclitaxel," Journal of Controlled Release, vol. 101, no. 1-3, pp. 59-68, 2005.

[49] M. H. M. Leung, H. Colangelo, and T. W. Kee, "Encapsulation of curcumin in cationic micelles suppresses alkaline hydrolysis," Langmuir, vol. 24, no. 11, pp. 5672-5675, 2008.

[50] Z. Ma, A. Haddadi, O. Molavi, A. Lavasanifar, R. Lai, and J. Samuel, "Micelles of poly(ethylene oxide)-b-poly( $\varepsilon$-caprolactone) as vehicles for the solubilization, stabilization, and controlled delivery of curcumin," Journal of Biomedical Materials Research-Part A, vol. 86, no. 2, pp. 300-310, 2008.

[51] J. Cortes and C. Saura, "Nanoparticle albumin-bound $\left(\right.$ nab $\left.^{\mathrm{TM}}\right)$-paclitaxel: improving efficacy and tolerability by targeted drug delivery in metastatic breast cancer," European Journal of Cancer, Supplement, vol. 8, no. 1, pp. 1-10, 2010.

[52] T. H. Kim, H. H. Jiang, Y. S. Youn et al., "Preparation and characterization of water-soluble albumin-bound curcumin nanoparticles with improved antitumor activity," International Journal of Pharmaceutics, vol. 403, no. 1-2, pp. 285-291, 2011.

[53] A. Mukerjee and J. K. Vishwanatha, "Formulation, characterization and evaluation of curcumin-loaded PLGA nanospheres 
for cancer therapy," Anticancer Research, vol. 29, no. 10, pp. 3867-3875, 2009.

[54] L. Mei, L. Chen, and L. Yang, "Preparation of curcumin-loaded microspheres and their sustained release performance," Journal of Fujian Medical University, vol. 44, no. 3, pp. 178$181,2010$.

[55] S. Manju and K. Sreenivasan, "Hollow microcapsules built by layer by layer assembly for the encapsulation and sustained release of curcumin," Colloids and Surfaces B, vol. 82, no. 2, pp. 588-593, 2011.

[56] Y. Wang, Z. Lu, H. Wu, and F. Lv, "Study on the antibiotic activity of microcapsule curcumin against foodborne pathogens," International Journal of Food Microbiology, vol. 136, no. 1, pp. 71-74, 2009.

[57] Z. Xiao-Hui, C. Yu-Xing, Z. Zi-Ming et al., "Pharmacokinetics of curcumin microcystin in rats," Chinese Journal of Experimental Traditional Medical Formulae, vol. 16, no. 2, pp. 107110, 2010.

[58] X. Wang, Y. Jiang, Y. W. Wang, M. T. Huang, C. T. Ho, and Q. Huang, "Enhancing anti-inflammation activity of curcumin through O/W nanoemulsions," Food Chemistry, vol. 108, no. 2, pp. 419-424, 2008.

[59] Y. M. Yin, F. D. Cui, C. F. Mu et al., "Docetaxel microemulsion for enhanced oral bioavailability: preparation and in vitro and in vivo evaluation," Journal of Controlled Release, vol. 140, no. 2, pp. 86-94, 2009.

[60] S. Setthacheewakul, S. Mahattanadul, N. Phadoongsombut, W. Pichayakorn, and R. Wiwattanapatapee, "Development and evaluation of self-microemulsifying liquid and pellet formulations of curcumin, and absorption studies in rats," European Journal of Pharmaceutics and Biopharmaceutics, vol. 76, no. 3, pp. 475-485, 2010.

[61] C. H. Liu, F. Y. Chang, and D. K. Hung, “Terpene microemulsions for transdermal curcumin delivery: effects of terpenes and cosurfactants," Colloids and Surfaces B, vol. 82, no. 1, pp. 63-70, 2011.

[62] C. C. Lin, H. Y. Lin, H. C. Chen, M. W. Yu, and M. H. Lee, "Stability and characterisation of phospholipid-based curcuminencapsulated microemulsions," Food Chemistry, vol. 116, no. 4, pp. 923-928, 2009.

[63] A. Barik, B. Mishra, A. Kunwar et al., "Comparative study of copper(II)-curcumin complexes as superoxide dismutase mimics and free radical scavengers," European Journal of $\mathrm{Me}$ dicinal Chemistry, vol. 42, no. 4, pp. 431-439, 2007.

[64] P. Zhang, Y. Liu, N. Feng, and J. Xu, "Preparation and evaluation of self-microemulsifying drug delivery system of oridonin," International Journal of Pharmaceutics, vol. 355, no. 1-2, pp. 269-276, 2008.

[65] E. M. M. Del Valle, "Cyclodextrins and their uses: a review," Process Biochemistry, vol. 39, no. 9, pp. 1033-1046, 2004.

[66] R. L. Carrier, L. A. Miller, and I. Ahmed, "The utility of cyclodextrins for enhancing oral bioavailability," Journal of Controlled Release, vol. 123, no. 2, pp. 78-99, 2007.

[67] A. L. Laza-Knoerr, R. Gref, and P. Couvreur, "Cyclodextrins for drug delivery," Journal of Drug Targeting, vol. 18, no. 9, pp. 645-656, 2010.

[68] E. I. Paramera, S. J. Konteles, and V. T. Karathanos, "Stability and release properties of curcumin encapsulated in Saccharomyces cerevisiae, $\beta$-cyclodextrin and modified starch," Food Chemistry, vol. 125, no. 3, pp. 913-922, 2011.

[69] V. R. Yadav, S. Prasad, R. Kannappan et al., "Cyclodextrincomplexed curcumin exhibits anti-inflammatory and antiproliferative activities superior to those of curcumin through higher cellular uptake," Biochemical Pharmacology, vol. 80, no. 7, pp. 1021-1032, 2010.

[70] M. M. Yallapu, M. Jaggi, and S. C. Chauhan, “ $\beta$-Cyclodextrincurcumin self-assembly enhances curcumin delivery in prostate cancer cells," Colloids and Surfaces B, vol. 79, no. 1, pp. 113-125, 2010.

[71] L. Xiang and L. Xiu-Li, "Influence of $\beta-\mathrm{CD}$ and its derivatives solubilization and fluoresence enhancement of curcumin," Chinese Journal of Pharmaceuticals, vol. 39, no. 3, pp. 194-199, 2008.

[72] Y. Liang, L. Ying-jie, S. Xiao-Feng et al., "Study on preparation of curcumin soluble agent," Journal of Tianjin University of Technology, vol. 25, no. 6, pp. 75-77, 2009.

[73] W. L. Chiou and S. Riegelman, "Pharmaceutical applications of solid dispersion systems," Journal of Pharmaceutical Sciences, vol. 60, no. 9, pp. 1281-1302, 1971.

[74] A. Paradkar, A. A. Ambike, B. K. Jadhav, and K. R. Mahadik, "Characterization of curcumin-PVP solid dispersion obtained by spray drying," International Journal of Pharmaceutics, vol. 271, no. 1-2, pp. 281-286, 2004.

[75] X. W. Huang, J. H. Xu, G. H. Wu, and C. X. Wen, "Pharmacokinetics of curcumin solid dispersion in mice," Chinese Pharmacological Bulletin, vol. 24, no. 11, pp. 1525-1527, 2008.

[76] H. Xiu-Wang, X. Jian-Hua, W. Cai-Xia et al., "Study on preparation and in vitro dissolution of curcumin-plasdone solid dispersion," Chinese Journal of Hospital Pharmacy, vol. 28, no. 21, pp. 1819-1822, 2008.

[77] Z. Li-Chao, Z. He-Ping, Q. Shan-Cong et al., "Improvement of dissolution of curcumin from curcumin-phospholipid complex using solid dispersion technique," Chinese Journal of Pharmaceuticals, vol. 39, no. 12, pp. 905-907, 2008.

[78] S. Onoue, H. Takahashi, Y. Kawabata et al., "Formulation design and photochemical studies on nanocrystal solid dispersion of curcumin with improved oral bioavailability," Journal of Pharmaceutical Sciences, vol. 99, no. 4, pp. 1871-1881, 2010.

[79] W. Wu, J. Shen, P. Banerjee, and S. Zhou, "Water-dispersible multifunctional hybrid nanogels for combined curcumin and photothermal therapy," Biomaterials, vol. 32, no. 2, pp. 598609, 2011.

[80] M. Ghosh, A. T. K. Singh, W. Xu, T. Sulchek, L. I. Gordon, and R. O. Ryan, "Curcumin nanodisks: formulation and characterization,” Nanomedicine, vol. 7, no. 2, pp. 162-167, 2010.

[81] L. Zheng and J. F. Song, "Curcumin multi-wall carbon nanotubes modified glassy carbon electrode and its electrocatalytic activity towards oxidation of hydrazine," Sensors and Actuators, B, vol. 135, no. 2, pp. 650-655, 2009. 

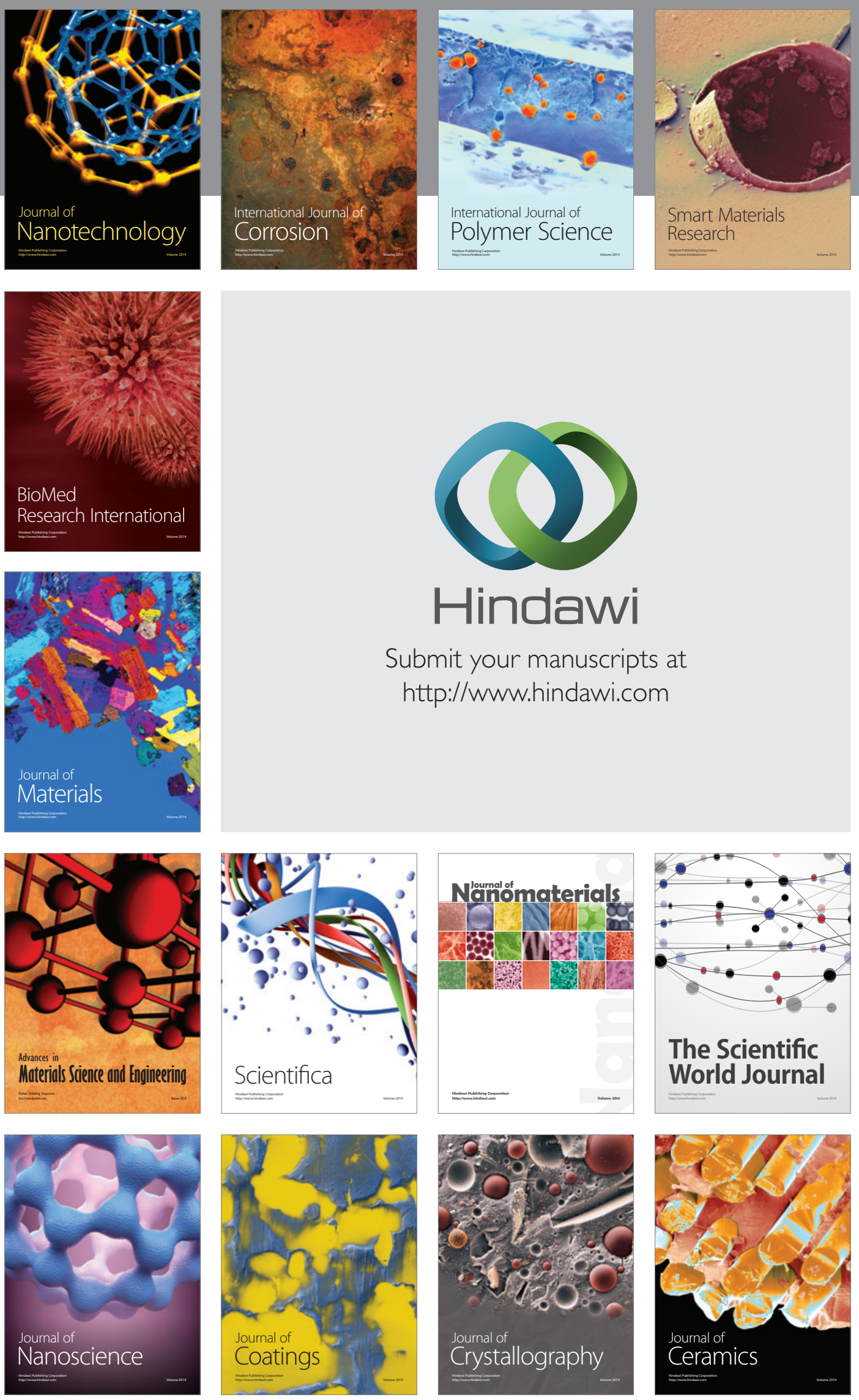

The Scientific World Journal

Submit your manuscripts at

http://www.hindawi.com

\section{World Journal}

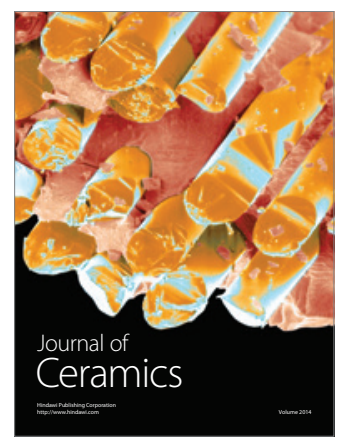

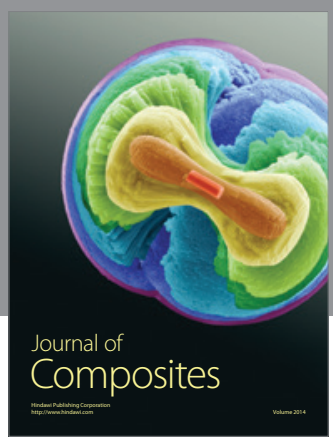
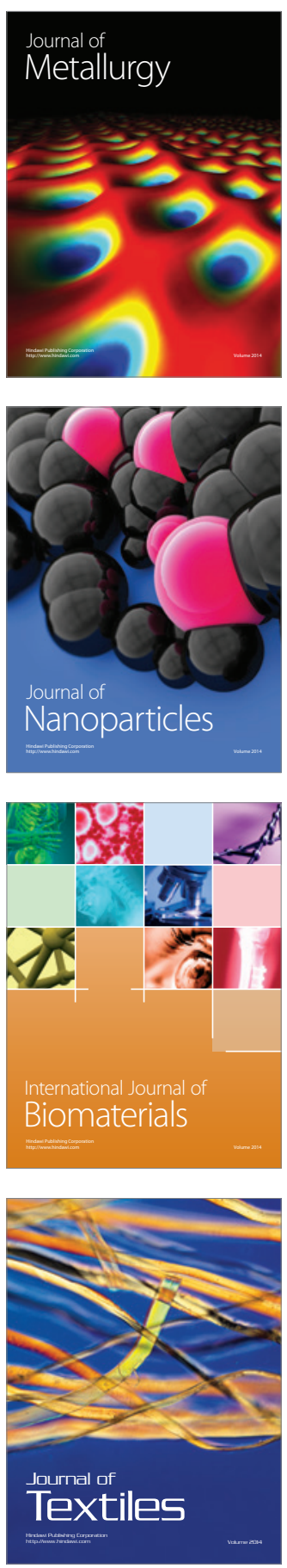